\title{
Functions of State for Spinor Gas in General Relativity
}

\author{
Yingqiu Gu \\ School of Mathematical Science, Fudan University, Shanghai, China \\ Email: yqgu@fudan.edu.cn
}

How to cite this paper: Gu, Y.Q. (2017) Functions of State for Spinor Gas in General Relativity. Open Access Library Journal, 4: e3953.

https://doi.org/10.4236/oalib.1103953

Received: September 20, 2017

Accepted: October 23, 2017

Published: October 26, 2017

Copyright (c) 2017 by author and Open Access Library Inc.

This work is licensed under the Creative Commons Attribution International License (CC BY 4.0).

http://creativecommons.org/licenses/by/4.0/

\begin{abstract}
The energy momentum tensor of perfect fluid is a simplified but successful model in astrophysics. In this paper, assuming the particles driven by gravity and moving along geodesics, we derived the functions of state in detail. The results show that, these functions have a little correction for the usual thermodynamics. The new functions naturally satisfy the causal condition and consist with relativity. For the self-potentials of the particles, we introduce an extra function $W$, which acts like negative pressure and can be used to describe dark matter. The results are helpful to understand the relation and interaction between space-time and matter.
\end{abstract}

Subject Areas

Mathematical Analysis, Particle Physics

\section{Keywords}

Energy Momentum Tensor, Equation of State, Geodesic, Self-Potential

\section{Introduction}

In astrophysics, the energy momentum tensor is usually described by perfect fluid model

$$
T^{\mu \nu}=(\rho+P) U^{\mu} U^{v}-P g^{\mu \nu}
$$

Theoretical analysis and experiments all show that (1.1) is a successful approximation. Some researchers such as Israel and Stewart [1] [2], Carter [3] and Lichnerowicz [4] disclose that, the energy momentum tensor $T^{\mu \nu}$ includes abundant contents for classical fluid theory, which includes not only energy and momentum, but also heat flux, spatial stress and viscosity [5] [6]. Furthermore, the relativistic hydrodynamics with variation principle and Cartan's exterior 
algebra was discussed in [7].

The cooling mechanism of the expanding universe is one of the most interesting problems of the students. The answers given by pedagogical articles [8] [9] [10] [11] are usually $T \propto a^{-1}$, which is derived from the classical thermodynamics. In [12] [13] [14] [15] [16], the author solved the thermodynamical relations according to the Gibbs' law

$$
\delta Q=\mathrm{d}(\rho V)+P \mathrm{~d} V
$$

The problem looks underdetermined due to (1.2) including a number of undetermined quantities. So we have to introduce some new relations such as multi fluid with energy exchange, the apparent horizon entropy, the decay of vacuum, to get a solution. As pointed out in [17], these approaches cannot convince the students and satisfy their curiosity. Dividing the velocity of particles into Hubble velocity $v_{h}=r a^{\prime}(t)$ and peculiar velocity $v_{p e c}=a r^{\prime}(t)$, and then analyzing the geodesic of a particle, the author qualitatively reached the conclusion $p \propto a^{-1}$. Then by mass-energy relation $E^{2}=p^{2}+m^{2}$, he concluded that the cosmic temperature should be $T \propto a^{-1}$ for the ultra-relativistic gas but $T \propto a^{-2}$ for the non-relativistic gas.

As a matter of fact, free particles in gravitational field will automatically move along geodesics, until they collide with each other. So the functions of state of gases consistent with general relativity should be naturally derived under background of gravity. This is the purpose of the paper. We derive the functions in microscopic point of view. From the calculation, indeed we can get complete functions of state, which automatically satisfy the requirement of relativity and remove the singularity from solutions to the Einstein's field equation [18].

\section{Ideal Gas in FRW Space-Time}

For dark spinors with self-interactive potential, in microscopic view, the energy momentum tensor can be expressed by [19]

$$
T^{\mu \nu}=\sum_{n}\left(m_{n} u_{n}^{\mu} u_{n}^{v}+w_{n} g^{\mu \nu}\right) \delta^{3}\left(\vec{x}-\vec{X}_{n}\right) \sqrt{1-v_{n}^{2}}
$$

where $m_{n}$ is the proper mass of the $n$-th spinor, $w_{n}>0$ is the proper potential of self-interaction, $u_{n}^{\mu} 4$-vector velocity, $\vec{v}_{n}$ the usual 3-d speed, $\vec{X}_{n}(t)$ the central coordinate. In the case of ideal gas, we have $w_{n}=0,(\forall n)$, and then we have the energy-momentum tensor (1.1) [8]. In case $w_{n}>0$, the complete average formalism of energy-momentum tensor should be

$$
T^{\mu \nu}=(\rho+P) U^{\mu} U^{v}+(W-P) g^{\mu \nu}
$$

where $W$ is a new function of state reflect the self-potential of particles, which acts like negative pressure and is defined by

$$
W=\frac{1}{V} \int_{V} \sum_{n} w_{n} \delta^{3}\left(\vec{x}-\vec{X}_{n}\right) \sqrt{1-v_{n}^{2}} \mathrm{~d} V=\frac{1}{V} \sum_{X_{n} \in V} w_{n} \sqrt{1-v_{n}^{2}}
$$

The functions of state in (2.1) and (2.3) including relativistic factor $\sqrt{1-v_{n}^{2}}$, 
which cannot calculate directly. We solve the problem by the following method, which naturally includes the interaction with gravity. To research the thermodynamical properties of gas, we use piston and cylinder to drive gas. In astrophysics, we have more ideal piston and cylinder that is the space-time with Friedmann-Robertson-Walker (FRW) metric, which is absolutely adiabatic and reversible. The FRW metric drives the gases homogeneously expanding and contracting as the scale factor a varies, and the results have general meanings according to the principle of equivalence.

In the microscopic view, for the ideal gases and photons, the particles are only driven by average gravity and move along geodesics, and the collisions between particles can be treated as instantaneous behavior. So all thermodynamic functions can be rigorously solved according to dynamics and statistics. In this section we set $w_{n}=0$ in (2.1) for simplicity.

For FRW space-time, we have the line element in conformal coordinate system

$$
\mathrm{d} s^{2}=a^{2}(t)\left(\mathrm{d} t^{2}-\mathrm{d} r^{2}-\mathcal{S}^{2}(r) \mathrm{d} \theta^{2}-S(r)^{2} \sin ^{2} \theta \mathrm{d} \varphi^{2}\right)
$$

where

$$
\mathcal{S}= \begin{cases}\sin r & \text { if } \kappa=1, \\ r & \text { if } \kappa=0, \\ \sinh r & \text { if } \kappa=-1\end{cases}
$$

The energy conservation law $T_{;, v}^{\mu v}$ in this case is equivalent to (1.2) while $\delta Q=0$, or manifestly

$$
\frac{\mathrm{d}\left(\rho a^{3}\right)}{\mathrm{d} a}=-3 P a^{2}
$$

For a given equation $\rho=\rho(a)$, we can solve the function $P=P(a)$ from (2.6) or vice versa.

To solve geodesic in FRW space-time, we have the following result.

Lemma 1. If the line element of the orthogonal subspace has the following form,

$$
\mathrm{d} s^{2}=A(t) \mathrm{d} t^{2}+\tilde{g}_{\mu \nu}(t) \mathrm{d} x^{\mu} \mathrm{d} x^{v}
$$

where $\boldsymbol{A}$ and $\tilde{g}_{\mu v}$ only depend on the coordinate $t$, then the geodesic in this subspace can be solved by

$$
\frac{\mathrm{d} x^{\mu}}{\mathrm{d} s}=\tilde{g}^{\mu v} C_{v}, \quad \frac{\mathrm{d} t}{\mathrm{~d} s}=\sqrt{\frac{1}{\boldsymbol{A}}\left(1-\tilde{g}^{\mu v} C_{\mu} C_{v}\right)}
$$

where $C_{\mu}$ are constants, and $\tilde{g}^{\mu v} \tilde{g}_{v \alpha}=\delta_{\alpha}^{\mu}$.

Lemma 1 can be checked directly. For the FRW metric (2.4), the line element in the orthogonal subspace $(t, r)$ is given by $\mathrm{d} s^{2}=a(t)^{2}\left(\mathrm{~d} t^{2}-\mathrm{d} r^{2}\right)$. According to Lemma 1, we have the geodesic equation

$$
\frac{\mathrm{d}}{\mathrm{d} s} r=\frac{C}{a^{2}}, \frac{\mathrm{d}}{\mathrm{d} s} t=\frac{1}{a^{2}} \sqrt{a^{2}+C^{2}}
$$


where $C$ is a constant only depends on the initial data. By (2.9) we get the drifting speed of a particle in usual sense

$$
v_{n} \equiv \frac{a \mathrm{~d} r}{a \mathrm{~d} t}=\frac{b_{n}}{\sqrt{a^{2}+b_{n}^{2}}}, \sqrt{1-v_{n}^{2}}=\frac{a}{\sqrt{a^{2}+b_{n}^{2}}}
$$

So the momentum of a particle $p=\frac{m_{n} v}{\sqrt{1-v^{2}}}$ satisfies

$$
p(t) a(t)=p\left(t_{0}\right) a\left(t_{0}\right)
$$

where $m_{n}$ is the proper mass of the particle. For the massless photons, we can check that the wavelength $\lambda(t)$ satisfies $\frac{\lambda(t)}{a(t)} \equiv \frac{\lambda_{0}}{a_{0}}$, so their momentum $p$ also satisfy (2.11). Although (2.11) is derived in subspace-time $(t, r)$, but it is suitable for all particles due to the symmetry of the FRW metric.

The relation between momentum $p$ and the kinetic energy $K$ is given by

$$
p^{2}=K(K+2 m)
$$

By (2.11) we have $p_{n}^{2}=\frac{C_{n}}{a^{2}}$, where $C_{n}$ are constants only depending on the initial data at $t=t_{0}$. Then on one hand, for all particles we have the mean square momentum directly

$$
\bar{p}^{2}=\frac{C_{0}}{a^{2}}
$$

where $C_{0}$ is a constant only determined by initial data at $t_{0}$. One may argue that (2.13) is probably broken by the collision of the particles. The following Lemma shows that (2.13) holds in statistical sense.

Lemma 2. The mean square momentum of the ideal gas is independent of the elastic collision of the particles.

Proof. For any elastic collision, we have momentum conservation law $\vec{p}_{1}+\vec{p}_{2}=\vec{P}_{1}+\vec{P}_{2}$, and then

$$
p_{1}^{2}+p_{2}^{2}=P_{1}^{2}+P_{2}^{2}+2\left(\vec{P}_{1} \cdot \vec{P}_{2}-\vec{p}_{1} \cdot \vec{p}_{2}\right)
$$

Taking average for (2.14), we have

$$
\bar{p}^{2}=\bar{P}^{2}+\Delta
$$

Since the elastic collision is a reversible process, in statistical sense, we have the exactly equal numbers of reversible process, so we also have

$$
\bar{P}^{2}=\bar{p}^{2}+\Delta
$$

Comparing (2.15) with (2.16), we have $\Delta=0$ and $\bar{p}^{2}=\bar{P}^{2}$. Since collision is finished instantaneously, (2.13) holds for all time $t$.

On the other hand, $\bar{p}^{2}$ can be calculated according to statistical principle. Assuming the distribution of kinetic energy $K$ of the particles is given by

$$
\mathrm{d} \mathcal{P}=\mathcal{F}(K) \mathrm{d} K
$$

then we have 


$$
\int_{0}^{\infty} \mathrm{d} \mathcal{P}=1, \int_{0}^{\infty} K \mathrm{~d} \mathcal{P}=\frac{3}{2} k T, \int_{0}^{\infty} K^{2} \mathrm{~d} \mathcal{P}=\frac{3}{2 \sigma}(k T)^{2}
$$

where the second formula can be regarded as definition of temperature, $\sigma$ is a constant reflecting the concrete distribution function of particles. In statistical mechanics, we usually use the distribution functions of momentum, which is inconvenient for calculation in the case of relativistic gases. Since the following discussions have nothing to do with explicit function $F(K)$, and at most uses the second order moment, so the kinetic energy distribution (2.17) is much convenient. In case of Maxwell distribution, we have

$$
\mathrm{d} \mathcal{P}=\exp \left(-\frac{K}{k T}\right) \sqrt{\frac{4 K}{\pi k T}} \frac{\mathrm{d} K}{k T}, \quad \sigma=\frac{2}{5}
$$

By the moments (2.18) we have

$$
\begin{aligned}
\bar{p}^{2} & =\sum_{n} \int_{0}^{\infty} \frac{1}{N} p_{n}^{2} \mathcal{F}\left(K_{n}\right) \mathrm{d} K_{n} \\
& =\sum_{n} \int_{0}^{\infty} \frac{1}{N} K_{n}\left(K_{n}+2 m_{n}\right) \mathcal{F}\left(K_{n}\right) \mathrm{d} K_{n} \\
& =\sum_{n} k T \frac{1}{N}\left(\frac{3}{2 \sigma} k T+3 m_{n}\right)
\end{aligned}
$$

where $N$ is the number of particles with mass $m_{n}$ in the volume $V=\Omega a^{3}$. Comparing (2.20) with (2.13), we get the equation of $T(a)$ as follows

$$
k T=\frac{\sigma \bar{m} b^{2}}{a\left(a+\sqrt{a^{2}+b^{2}}\right)}, \quad a=\frac{\sigma \bar{m} b}{\sqrt{k T(k T+2 \sigma \bar{m})}}
$$

where $\bar{m}=\frac{1}{N} \sum_{n} m_{n}$ is the average mass of all particles, and $b$ is a constant only depending on initial data. Solving (2.21), we get

Theorem 3. The temperature of ideal gases in FRW space-time satisfies

$$
k T=\frac{\sigma \bar{m} b^{2}}{a\left(a+\sqrt{a^{2}+b^{2}}\right)}=\sigma \bar{m}\left(\sqrt{1+\frac{b^{2}}{a^{2}}}-1\right)
$$

where $b$ is constant determined by the initial data $a_{0}$ and $T_{0} \cdot a(t)$ acts as intermediate parameter.

By the theorem we find that, the cosmic temperature is different from the results directly derived from classical thermodynamics. In what follows we derive the relations between $\rho$ and $T$ as well as the equation of state.

In microscopic point of view, the Lagrangian of FRW space-time coupling with particles is given by [8] [20]

$$
\mathcal{L}=\frac{1}{16 \pi G}(R-2 \Lambda)-\sum_{n} m_{n} \sqrt{1-v_{n}^{2}} \delta^{3}\left(\vec{x}-\vec{X}_{n}\right)
$$

where $\vec{X}_{n}$ is the coordinate of $n$-th particle, the scalar curvature

$$
R=6 \frac{a^{\prime \prime}+\kappa a}{a^{3}}
$$


in which the prime stands for $\frac{\mathrm{d}}{\mathrm{d} t}$, and the drifting speed of $n$-th particle in usual sense

$$
\vec{v}_{n}=\left(v_{r}, v_{\theta}, v_{\varphi}\right)_{n}=\left(\frac{a \mathrm{~d} r}{a \mathrm{~d} t}, \frac{a \mathcal{S} \mathrm{d} \theta}{a \mathrm{~d} t}, \frac{a \mathcal{S} \sin \theta \mathrm{d} \varphi}{a \mathrm{~d} t}\right)_{n}
$$

are independent variables related to a for variation. Noticing that $\delta^{3}\left(\vec{x}-\vec{X}_{n}\right) \propto a^{-3}$, by variation of $I=\int \mathcal{L} a^{4} \mathrm{~d} t \mathrm{~d} \Omega$ with respect to $a$, we get

$$
a^{\prime \prime}+\kappa a-\frac{2}{3} \Lambda a^{3}=\frac{4 \pi G}{3 \Omega} \sum_{X_{n} \in \Omega} m_{n} \sqrt{1-v_{n}^{2}}
$$

where $\Omega$ is any given comoving volume with volume element $\mathrm{d} \Omega=\mathcal{S}^{2} \sin \theta \mathrm{d} r \mathrm{~d} \theta \mathrm{d} \varphi$, which is independent of a. Substituting (2.10) into (2.25), we get

$$
a^{\prime \prime}+\kappa a-\frac{2}{3} \Lambda a^{3}=\frac{4 \pi G}{3 \Omega} \sum_{X_{n} \in \Omega} \frac{m_{n} a}{\sqrt{a^{2}+b_{n}^{2}}}
$$

Multiply (2.26) by $a^{\prime}$ and integrate it, again by (2.10) we have

$$
\begin{aligned}
a^{\prime 2}+\kappa a^{2}-\frac{1}{3} \Lambda a^{4} & =\frac{8 \pi G}{3 \Omega} \sum_{X_{n} \in \Omega} \frac{m_{n} a}{\sqrt{1-v_{n}^{2}}}+C_{1} \\
& =\frac{8 \pi G}{3 \Omega} \sum_{X_{n} \in \Omega}\left(K_{n}+m_{n}\right) a+C_{1}
\end{aligned}
$$

where $C_{1}$ is a constant, for classical particles $C_{1}=0 \quad$ [20].

By Friedmann equation and (2.22), making statistical average of (2.27) we get

$$
\begin{aligned}
\frac{8 \pi G}{3} \rho a^{4} & =a^{\prime 2}+\kappa a^{2}-\frac{1}{3} \Lambda a^{4} \\
& =\frac{8 \pi G}{3} \bar{\rho}\left(1+\frac{3}{2} \frac{\sigma b^{2}}{a\left(a+\sqrt{a^{2}+b^{2}}\right)}\right) a^{4}
\end{aligned}
$$

where $\rho$ is defined by (1.1) and $\bar{\rho}$ by the following

$$
\rho=\frac{1}{V} \sum_{X_{n} \in V} E_{n}=\frac{1}{V} \sum_{X_{n} \in V}\left(K_{n}+m_{n}\right), \quad \bar{\rho}=\frac{1}{V} \sum_{X_{n} \in V} m_{n}=\frac{\varrho}{a^{3}}
$$

in which $\varrho=\frac{1}{\Omega} \sum_{n} m_{n}$ is the comoving density independent of a. Comparing (2.28) with (2.21), we get

Theorem 4. For the ideal gas in FRW space-time, the mass density satisfies

$$
\begin{gathered}
\rho=\frac{\rho}{a^{3}}\left(1+\frac{3 \sigma}{2 a}\left(\sqrt{a^{2}+b^{2}}-a\right)\right)=\bar{\rho}\left(1+\frac{3}{2} \frac{k T}{\bar{m}}\right) \\
\bar{\rho}=\rho_{0}[k T(k T+2 \sigma \bar{m})]^{\frac{3}{2}}
\end{gathered}
$$

where $\rho_{0}$ is a constant.

Substituting (2.30) into (2.6), we get

Theorem 5. The equation of state for ideal gas in FRW space-time is given by 


$$
\begin{aligned}
P & =\frac{\sigma \varrho b^{2}}{2 a^{4} \sqrt{a^{2}+b^{2}}}=\frac{N k T}{V}\left(1-\frac{k T}{2(\sigma \bar{m}+k T)}\right) \\
& =\frac{\varrho_{0}[k T(k T+2 \sigma \bar{m})]^{\frac{5}{2}}}{2 \bar{m}(k T+\sigma \bar{m})}=\frac{\bar{\rho} k T(k T+2 \sigma \bar{m})}{2 \bar{m}(k T+\sigma \bar{m})}
\end{aligned}
$$

The final expressions of function of state (2.30) and (2.32) are independent of metric a which is only used as "piston-cylinder" to drive the particles. So the state functions (2.30) and (2.32) are generally valid for ideal gases in general relativity.

Denoting $J=\frac{k T}{\bar{m} c^{2}}$, by (2.30) and (2.32) we have asymptotic properties of equation of state (EOS) for the particles

$$
P \doteq \begin{cases}P_{0} \rho^{\frac{5}{3}}\left(1-\frac{1}{2 \sigma}(5 \sigma+2) J\right) & \text { if } T \rightarrow 0, \\ \frac{1}{3} \rho\left(1+\left(\sigma-\frac{2}{3}\right) J^{-1}\right) & \text { if } T \rightarrow \infty\end{cases}
$$

The velocity of sound

$$
C_{s} \equiv c \sqrt{\frac{\mathrm{d} P}{\mathrm{~d} \rho}}=\frac{\sqrt{3}}{3}\left(\frac{c^{2} J(2 \sigma+J)\left(5 \sigma^{2}+8 \sigma J+4 J^{2}\right)}{(\sigma+J)^{2}\left[2 \sigma+(2+5 \sigma) J+4 J^{2}\right]}\right)^{\frac{1}{2}}<\frac{\sqrt{3}}{3} c
$$

Obviously the EOS satisfies the increasing and causal conditions which are consistent with relativity.

\section{Functions of State Including Self-Potentials}

In this section, we consider the case $w_{n} \neq 0$ in (2.1). By (2.10), we have relation

$$
\sqrt{1-v_{n}^{2}}=\frac{\mathrm{d}}{\mathrm{d} a} \frac{a}{\sqrt{1-v_{n}^{2}}}=\frac{1}{m_{n}} \frac{\mathrm{d}}{\mathrm{d} a}\left[a\left(K_{n}+m_{n}\right)\right]
$$

This is the key relation to calculate the relativistic factor $\sqrt{1-v_{n}^{2}}$. Substituting it into (2.21), and using (2.22) and (2.18), we get

$$
\begin{gathered}
\sum_{n} \frac{m_{n}}{N} \int \sqrt{1-v_{n}^{2}} \mathrm{~d} \mathcal{P}=\frac{\mathrm{d}}{\mathrm{d} a}\left(\frac{a}{N} \sum_{n} \int\left(K_{n}+m_{n}\right) \mathrm{d} \mathcal{P}\right)=\bar{m}-\frac{3 \sigma \bar{m} k T}{2(\sigma \bar{m}+k T)} \\
\sum_{n} \frac{w_{n}}{N} \int \sqrt{1-v_{n}^{2}} \mathrm{~d} \mathcal{P}=\frac{\mathrm{d}}{\mathrm{d} a}\left(\frac{a}{N} \sum_{n} \frac{w_{n}}{m_{n}} \int\left(K_{n}+m_{n}\right) \mathrm{d} \mathcal{P}\right)=\bar{w}-\frac{3 \sigma \bar{\mu} \bar{m} k T}{2(\sigma \bar{m}+k T)}
\end{gathered}
$$

where the mean parameters are defined by

$$
\bar{w}=\frac{1}{N} \sum_{n}^{N} w_{n}, \quad \bar{\mu}=\frac{1}{N} \sum_{n}^{N} \frac{w_{n}}{m_{n}}
$$

In the case $w_{n}>0$, by (3.3) we have

$$
\sum_{n} w_{n} \int \sqrt{1-v_{n}^{2}} \mathrm{~d} \mathcal{P}>0, \quad \sigma \bar{\mu}<\frac{2 \bar{w}}{3 \bar{m}}
$$


For the same kind particles $\bar{w}=\bar{\mu} \bar{m}$, we find $\sigma<\frac{2}{3}$. If the scale of the space-time is stable and varies slowly, e.g. in a galaxy, all the proper parameters such as $w_{n}$ and $m_{n}$ can be treated as constants.

If we define the mass-energy density $\rho$ and pressure $P$ of the particles from (2.1) in micro form as usual [8]

$$
\rho \equiv \sum_{n} \frac{m_{n}}{\sqrt{1-v_{n}^{2}}} \delta^{3}\left(\vec{x}-\vec{X}_{n}\right), \quad P \equiv \frac{1}{3} \sum_{n} \frac{m_{n} v_{n}^{2}}{\sqrt{1-v_{n}^{2}}} \delta^{3}\left(\vec{x}-\vec{X}_{n}\right)
$$

then in mean sense we get

$$
\rho=\frac{1}{V} \int \mathrm{d} \mathcal{P} \int_{V} \sum_{n}\left(K_{n}+m_{n}\right) \delta^{3}\left(\vec{x}-\vec{X}_{n}\right) \mathrm{d} V=\bar{\rho}\left(1+\frac{3 k T}{2 \bar{m}}\right)
$$

Again we get (2.30). By (3.2) and (3.6), we have

$$
P=\frac{1}{3 V} \int \mathrm{d} \mathcal{P} \sum_{X_{n} \in V}\left(\frac{m_{n}}{\sqrt{1-v_{n}^{2}}}+m_{n} \sqrt{1-v_{n}^{2}}\right)=\bar{\rho} \frac{k T}{2 \bar{m}} \frac{2 \sigma \bar{m}+k T}{\sigma \bar{m}+k T}
$$

Again we get (2.32). Similarly, by (2.3) and (3.3) we get $W$ in average form

$$
W=\frac{1}{V} \int \sum_{X_{n} \in V} w_{n} \sqrt{1-v_{n}^{2}} \mathrm{~d} \mathcal{P}=\bar{\rho}\left(\frac{\bar{w}}{\bar{m}}-\frac{3 \bar{\mu} \sigma k T}{2(\sigma \bar{m}+k T)}\right)
$$

The equations of state (3.7), (3.8) and (3.9) are valid for any ideal gases in local equilibrium. They have the following dimensionless form,

$$
\begin{gathered}
J \equiv \frac{k T}{\bar{m} c^{2}}, \quad \bar{\rho}=\varrho_{0}[J(J+2 \sigma)]^{\frac{3}{2}} \\
\rho=\bar{\rho}\left(1+\frac{3}{2} J\right)=\varrho_{0}[J(J+2 \sigma)]^{\frac{3}{2}}\left(1+\frac{3}{2} J\right) \\
P=\bar{\rho} \frac{J(J+2 \sigma)}{2(J+\sigma)}=\varrho_{0}[J(J+2 \sigma)]^{\frac{5}{2}} \frac{1}{2(J+\sigma)} \\
W=\bar{\rho}\left(\mu-\frac{3 \bar{\mu} \sigma J}{2(J+\sigma)}\right)=\varrho_{0}[J(J+2 \sigma)]^{\frac{3}{2}}\left(\mu-\frac{3 \bar{\mu} \sigma J}{2(J+\sigma)}\right)
\end{gathered}
$$

where $\rho_{0}$ is a constant depending on parameters $(\bar{m}, b, \sigma, \mu, \bar{\mu})$, and $\mu=\frac{\bar{w}}{\bar{m}}$. Among the functions of state only the temperature $k T$ or $J$ is independent variable.

Equations (3.10)-(3.13) are based on the assumption that the particles move along geodesic. This is valid for $0 \leq w_{n} \ll m_{n}$. In general cases, these functions can be modified by the following treatments. In (3.10)-(3.13), we take density $\bar{\rho}$ or equivalently the volume $V$ as an independent state function, and then derive the function $\bar{\rho}(J)$ according to energy conservation law.

In the comoving coordinate system with the following Gaussian type metric [8] [21]

$$
g_{\mu \nu}=\operatorname{diag}\left(g_{00},-\tilde{g}_{a b}\right), \quad(a, b) \in\{1,2,3\}
$$


where $\tilde{g}_{a b}$ is the spatial metric, for energy-momentum tensor (2.2), we have

Theorem 6. For the particles with energy-momentum tensor (2.2), we have the following Gibbs-Duhem's law

$$
\delta Q=\mathrm{d}[(\rho+W) V]+(P-W) \mathrm{d} V
$$

where $\delta Q$ denotes the heat received by the $N$ particles, and

$$
V=\sqrt{\tilde{g}} \Delta x \Delta y \Delta z, \quad\left(\tilde{g}=\operatorname{det}\left(\tilde{g}_{a b}\right)\right)
$$

is the micro spatial volume occupied by $N$ given particles.

This can be checked as follows. We trace the motion of these particles. In the adiabatic process, we have $\delta Q=0$. Then by $U_{\mu} T_{; v}^{\mu v}=0$, we get the continuity equation for (2.2) as

$$
U^{\mu} \partial_{\mu}(\rho+W)+(\rho+P) U_{; \mu}^{\mu}=0
$$

Denoting the proper time by $\mathrm{d} \tau$, then $\frac{\mathrm{d}}{\mathrm{d} \tau}=U^{\mu} \partial_{\mu}$, (3.17) becomes

$$
\begin{aligned}
0= & \sqrt{|g|} \frac{\mathrm{d}}{\mathrm{d} \tau}(\rho+W)+(\rho+P)\left(\sqrt{|g|} \partial_{\mu} U^{\mu}+\frac{\mathrm{d}}{\mathrm{d} \tau} \sqrt{|g|}\right) \\
= & \sqrt{g_{00}}\left(\frac{\mathrm{d}}{\mathrm{d} \tau}((\rho+W) \sqrt{\tilde{g}})+(P-W) \frac{\mathrm{d}}{\mathrm{d} \tau} \sqrt{\tilde{g}}\right) \\
& +(\rho+P) \sqrt{\tilde{g}}\left(\sqrt{g_{00}} \partial_{\mu} U^{\mu}+\frac{\mathrm{d}}{\mathrm{d} \tau} \sqrt{g_{00}}\right)
\end{aligned}
$$

where $g=\operatorname{det}\left(g_{\mu v}\right)=-g_{00} \tilde{g}$. In the comoving system, we have $U^{\mu}=\left(\sqrt{g^{00}}, 0,0,0\right)$ and $\mathrm{d} \tau=\sqrt{g_{00}} \mathrm{~d} t$, then we get

$$
\sqrt{g_{00}} \partial_{\mu} U^{\mu}+\frac{\mathrm{d}}{\mathrm{d} \tau} \sqrt{g_{00}}=\sqrt{g_{00}} \partial_{t} \frac{1}{\sqrt{g_{00}}}+\frac{1}{\sqrt{g_{00}}} \partial_{t} \sqrt{g_{00}}=0
$$

Multiplying (3.18) by the comoving volume element $\Delta x \Delta y \Delta z$ for the $N$ given particles, (3.18) gives (3.15) in the case $\delta Q=0$. In the case of $\delta Q \neq 0$, (3.15) holds due to energy conservation law.

Now we derive the relation $\bar{\rho}(J)$ from (3.15) for the equilibrium process with $\delta Q=0$. For clearness, we use the the static mass density $\bar{\rho}=\frac{N \bar{m}}{V}$ to replace $V$. Substituting (3.7), (3.8) and (3.9) into (3.15), we get dimensionless differential equation

$$
\frac{1}{\bar{\rho}} \frac{\mathrm{d} \bar{\rho}}{\mathrm{d} J}=\frac{3\left[(J+\sigma)^{2}-\bar{\mu} \sigma^{2}\right]}{(J+\sigma)\left(J^{2}+B J-2 \mu \sigma\right)}
$$

The solution is given by

$$
\bar{\rho}=\varrho_{0}(J+\sigma)^{\frac{3 \bar{\mu}}{3 \bar{\mu}+1}}\left(J^{2}+J B-2 \sigma \mu\right)^{\frac{3}{2}\left(1-\frac{\bar{\mu}}{3 \bar{\mu}+1}\right)}\left(\frac{2 J+B-A}{2 J+B+A}\right)^{\frac{\alpha}{2 A}}
$$

where parameters are defined by 


$$
B=(2+3 \bar{\mu}) \sigma-2 \mu, \quad A=\sqrt{8 \sigma \mu+B^{2}}, \quad \alpha=3(1+4 \bar{\mu})(2 \mu-3 \bar{\mu} \sigma)
$$

(3.21) shows how the internal potentials $w_{n}$ influence the mass density. (3.21) reduces to (3.10) if $\mu=\bar{\mu}=0$.

\section{Discussion and Conclusion}

1) The above calculations show that functions of state consistent with relativity should include the influences of gravity. The energy-momentum tensor and geodesics connect the macro concepts with micro movements of particles.

2) In the case of ideal gas with $\bar{w}=0$ at low temperature, (3.11)-(3.12) give the equation of state for the adiabatic monatomic gas

$$
P \doteq \rho J \doteq P_{0} \rho^{\frac{5}{3}}, \quad(J \ll 1 \text { or } k T \ll \bar{m})
$$

which is identical to the empirical law in thermodynamics. When $J \gg 1$, we have $P \rightarrow \frac{1}{3} \rho$, so the adiabatic index is not a constant for large range of temperature due to the relativistic effect. These results show the validity of (3.11)-(3.13) and the consistence with normal thermodynamics.

3) By (3.11), letting $J \rightarrow \infty$ or $\bar{m} \rightarrow 0$, we get the Stefan-Boltzmann's law $\rho \propto T^{4}$. This means that the above results automatically include photons, and the Stefan-Boltzmann's law is also valid for the ultra-relativistic particles.

4) In general relativity, all processes occur automatically, and $\varrho_{0}$ is independent of any practical process. Of course, $\varrho_{0}$ is related to the property of particles. Furthermore, equation of state (3.11)-(3.12) provides a singularity-free stellar structure in thermal equilibrium [18].

$5)$ In the case $\bar{w}>0$, the motion of the particles will slightly deviate from the geodesic. By (3.21) we find, $\bar{\rho}=0$ leads to $J=\frac{2 \sigma \mu}{B} \approx \mu$, which means the zero temperature can not reach. The physical reason for such conclusion is unclear.

\section{Acknowledgements}

The author is grateful to Prof. Ta-Tsien, Prof. Tie-Hu Qin and Prof. Ji-Zong Li for encouragement.

\section{References}

[1] Stewart, G.J.M. (1977) On Transient Relativistic Thermodynamics and Kinetic Theory. Proceedings of the Royal Society London A, 357, 59-75. https://doi.org/10.1098/rspa.1977.0155

[2] Israel, W. and Stewart, J.M. (1979) Transient Relativistic Thermodynamics and Kinetic Theory. Annals of Physics, 118, 341-372. https://doi.org/10.1016/0003-4916(79)90130-1

[3] Carter, B. (1991) Convective Variational Approach to Relativistic Thermodynamics of Dissipative Fluids. Proceedings of the Royal Society London A, 433, 45. https://doi.org/10.1098/rspa.1991.0034 
[4] Lichnerowicz, A. (1967) Relativistic Hydrodynamics and Magnetohydrodynamics. Benjamin, New York.

[5] Andersson, N. and Comer, G.L. (2007) Relativistic Fluid Dynamics: Physics for Many Different Scales. Living Reviews in Relativity, 10, 1.

[6] Hiscock, W.A. and Lindblom, L. (1983) Stability and Causality in Dissipative Relativistic Fluids. Annals of Physics, 151, 466-496.

https://doi.org/10.1016/0003-4916(83)90288-9

[7] Gourgoulhon, E. (2006) An Introduction to Relativistic Hydrodynamics. EAS Publications Series, 21, 43. https://doi.org/10.1051/eas:2006106

[8] Weinberg, S.L. (1972) Gravitation and Cosmology. Ch. 2.8, Ch. 5, Ch. 11, Wiley, New York.

[9] Burko, L.M. (1995) Answer to Question \#10, Cooling and Expansion of the Universe. American Journal of Physics, 63, 1065-1066. https://doi.org/10.1119/1.18006

[10] Keeports, D. (1995) Answer to Question \#10, Cooling and Expansion of the Universe. American Journal of Physics, 63, 1067.

[11] Blau, S. (1995) What Happens to Energy in the Cosmic Expansion? American Journal of Physics, 63, 1066-1067. https://doi.org/10.1119/1.18007

[12] Clifton, T. and Barrow, J.D. (2007) The Ups and Downs of Cyclic Universes. Physical Review D, 75, Article ID: 043515. https://doi.org/10.1103/PhysRevD.75.043515

[13] Gong, Y.G., Wang, B. and Wang, A.Z. (2007) Thermodynamical Properties of the Universe with Dark Energy. JCAP, 0701, 024. https://doi.org/10.1088/1475-7516/2007/01/024

[14] Alcaniz, J.S.J. and Lima, A.S. (2005) Interpreting Cosmological Vacuum Decay. Physical Review D, 72, Article ID: 063516. https://doi.org/10.1103/PhysRevD.72.063516

[15] Izquierdo, G. and Pavón, D. (2006) Dark Energy and the Generalized Second Law. Physics Letters B, 633, 420-426.

[16] Clifton, T. and Barrow, J.D. (2006) Decaying Gravity. Physical Review D, 73, Article ID: 104022. https://doi.org/10.1103/PhysRevD.73.104022

[17] Rahvar, S. (2006) Cooling in the Universe.

[18] Gu, Y.Q. (2007) Structure of the Star with Ideal Gases.

[19] Gu, Y.Q. (2017) The Vierbein Formalism and Energy-Momentum Tensor of Spinors.

[20] Gu, Y.Q. (2007) A Cosmological Model with Dark Spinor Source. International Journal of Modern Physics A, 22, 4667-4678. https://doi.org/10.1142/S0217751X07037925

[21] Gu, Y.Q. (2017) Natural Coordinate System in Curved Space-Time. 
Submit or recommend next manuscript to OALib Journal and we will provide best service for you:

- Publication frequency: Monthly

- 9 subject areas of science, technology and medicine

- Fair and rigorous peer-review system

- Fast publication process

- Article promotion in various social networking sites (LinkedIn, Facebook, Twitter, etc.)

- Maximum dissemination of your research work

Submit Your Paper Online: Click Here to Submit

Or Contact service@oalib.com 